\title{
Efficacy of $N$-acetylcysteine and all-trans retinoic acid in restoring in vitro effective hemopoiesis in myelodysplastic syndromes
}

\author{
Agostino Cortelezzi a,*, Chiara Cattaneo a, Barbara Sarina a, Silvia Cristiani a, \\ Mauro Pomati a, Ilaria Silvestris ${ }^{\text {, }}$, Marina Motta ${ }^{\text {a, Adalberto Ibatici a }}$, \\ Gianluca Gornati ${ }^{b}$, Aldo Della Volpe ${ }^{\mathrm{c}}$, Anna Teresa Maiolo ${ }^{\mathrm{a}}$ \\ a Servizio Autonomo di Ematologia Diagnostica, Ospedale Maggiore Policlinico IRCCS, Via F. Sforza 35, 20122 Milan, Italy \\ ${ }^{\mathrm{b}}$ Centro Trasfusionale, Istituti Clinici di Perfezionamento, Milan, Italy \\ ${ }^{\mathrm{c}}$ Centro Trapianti di Midollo, Ospedale Maggiore Policlinico IRCCS, Milan, Italy
}

Received 26 April 1999; accepted 20 August 1999

\begin{abstract}
We evaluated the in vitro effect on clonogenic potential (CFU-GM) and apoptosis in myelodysplastic syndromes (MDS) progenitors of an anti-oxidant ( $N$-acetylcysteine, NAC) and/or a differentiating (all-trans retinoic acid, ATRA) agent. NAC significantly reduced apoptosis, both NAC and ATRA induced an increase in CFU-GM, but NAC seemed to be particularly effective in the high risk (HR) MDS. NAC + ATRA conferred a significant advantage in terms of CFU-GM with respect to NAC and ATRA alone. Tumor Necrosis Factor- $\alpha$ (TNF- $\alpha$ ) levels decreased after incubation with NAC in the MDS samples. This study shows that ineffective hemopoiesis in MDS could benefit from both NAC and ATRA, suggesting that anti-oxidant treatment may play a role in guaranteeing MDS cell survival, predisposing them towards differentiation. (C) 2000 Published by Elsevier Science Ltd. All rights reserved.
\end{abstract}

Keywords: Myelodysplastic syndromes; $N$-acetylcysteine; All-trans retinoic acid; Apoptosis; Clonogenic activity

\section{Introduction}

Increased bone marrow apoptosis and ineffective hemopoiesis are the two main characteristics of the myelodysplastic syndromes (MDS) [1-6]. The former, which is at least partially responsible for premature intramedullary cell death, is particularly pronounced in low-risk MDS (such as refractory anemia, RA and refractory anemia with ring sideroblasts, RARS), whereas the latter leads to the well known maturation arrest of bone marrow (BM) progenitors, but both

Abbreviations: ATRA, all-trans-retinoic acid; BM, bone marrow; BMMNC, bone marrow mononuclear cell; HR, high risk; LR, low risk; MDS, myelodysplastic syndromes; NAC, $N$-acetylcysteine; RA, refractory anemia; RAEB, refractory anemia with excess of blasts; RAEB-t, refractory anemia with excess of blasts in transformation; RARS, refractory anemia with ring sideroblasts; TNF- $\alpha$, tumor necrosis factor- $\alpha$.

* Corresponding author. Tel.: + 39-02-5503-3429/3345; fax: + 3902-5503-3380.

E-mail address: cortelez@polic.cilea.it (A. Cortelezzi) determine the often severe and life-threatening cytopenia(s) typical of MDS.

It is well documented that MDS patients have increased serum Tumor Necrosis Factor- $\alpha$ (TNF- $\alpha$ ) levels [7-11], which may be responsible for pro-apoptotic oxidative damage by inducing the generation of free radicals [12-16]. In line with this hypothesis, Peddie et al. [17] have demonstrated the presence of oxidative damage and a reduction in intracellular glutathione $(\mathrm{GSH})$ in MDS CD34 + cells, and a number of in vitro studies have shown the ability of the antioxidant thiol $\mathrm{N}$-acetylcysteine (NAC) to reduce the apoptosis induced by free radicals in neuronal and leukemic cell lines by increasing the supply of intracellular GSH [18-20]. NAC also acts as an antiapoptotic agent by inhibiting TNF- $\alpha$ release from lymphocytes and accessory cells [21].

MDS bone marrow (BM) progenitors can be induced to differentiate and mature in vitro by using various differentiating agents, such as vitamin $\mathrm{D}_{3}$ and $\mathrm{E}$, Ara- 
$\mathrm{C}$, butyrates and amifostine and this has provided the rationale for several clinical trials [22].

ATRA is a natural metabolite of retinol, whose differentiating activity has also been demonstrated in non-APL blast cells [23]. However, when used in vivo, the ability of ATRA alone to restore defective hemopoiesis in MDS has not always been demonstrated [24-30].

On the basis of these observations we incubated MDS progenitor cells in vitro first with NAC alone and after $24 \mathrm{~h}$ with ATRA alone or in combination in order to explore their activity in terms of enhanced clonogenic potential (CFU-GM), reduced apoptosis and stimulated differentiation. The rationale of the sequence (first NAC then ATRA) was based on the hypothesis of first reducing the degree of apoptosis by means of an antioxidant, and then stimulating differentiation via ATRA. The possible role of TNF- $\alpha$ in inducing free radicals was evaluated by determining TNF- $\alpha$ levels in bone marrow plasma and in supernatants of liquid cultures.

\section{Patients}

The BM cells were obtained from 25 patients with de novo MDS (21 males and 4 females, with a median age of 68 years: range 34-86) and seven normal bone marrow donors, after they had given their informed consent. On the basis of the FAB criteria, the patients were classified as being affected by RA $(n=6)$, RARS $(n=7)$, RAEB $(n=10)$ and RAEB-t $(n=2)$. For the purpose of the study, we classified RA + RARS as low risk (LR) MDS and RAEB + RAEB-t as high risk (HR) MDS.

\section{Methods}

\subsection{Cell preparation}

The BM samples were collected in preservative-free heparin. The mononuclear cells (MNCs) were separated by means of gradient centrifugation using Ficoll Lymphoprep (Nicomed Pharma Oslo, Norway), evaluated for apoptosis and then suspended in IMDM (Gibco Europe, Paisley, UK) with 10\% FCS (Hyclone, Logan, Utah, USA ) at a concentration of $1 \times 10^{6} / \mathrm{ml}$ and incubated at $37^{\circ} \mathrm{C}$ in $5 \% \mathrm{CO}_{2} \pm \mathrm{NAC} 0.5 \mathrm{mM}$ for $24 \mathrm{~h}$. After this time, some of the cells \pm NAC (Sigma, Saint Louis, MO, USA) were evaluated for CFU-GM and apoptosis and others were cultured \pm ATRA $5 \mu \mathrm{M}$ (kindly provided by Roche) for a further $24 \mathrm{~h}$. After a total culture time of $48 \mathrm{~h}$, the cells incubated with ATRA, NAC, NAC + ATRA as well as the control cells were again tested for CFU-GM and apoptosis.
Due to the scanty cell recovery from MDS BM, the samples incubated for $48 \mathrm{~h}$ with NAC alone were evaluated in only $12 / 25$ cases. The viability after liquid incubation was always $>90 \%$ evaluated by the Trypan blue exclusion test.

\subsection{Apoptosis}

Apoptosis was evaluated by means of a TdT/dUTP assay using the commercially available 'In Situ Cell Death Fluorescein Detection Kit' (Boehringer Mannheim, Germany). Briefly, $1-2 \times 10^{6}$ cells were fixed in cold ethanol $70 \%$ in PBS, and stored at $4^{\circ} \mathrm{C}$ until use. Before staining, the cells were washed in PBS, permeabilized with $100 \mu \mathrm{l}$ TritonX-100 $0.1 \%$ in sodium citrate $0.1 \%$ for $2 \mathrm{~min}$ on ice, and again washed twice in PBS. After incubation for $1 \mathrm{~h}$ at $37^{\circ} \mathrm{C}$ with dUTP FITC, with and without TdT, samples were again washed in PBS, resuspended in $500 \mu 1$ of PBS and made ready for cytofluorimetric analysis. A total of $1 \times 10^{4}$ events were analyzed.

\section{3. $C F U-G M$}

A volume of $1 \times 10^{5}$ cells $/ \mathrm{ml}$ in IMDM were cultured in $1 \mathrm{ml}$ of a mixture containing $20 \% \mathrm{FCS}, 0.3 \%$ agar, GM-CSF $200 \mathrm{U} / \mathrm{ml}$, IL-3 $100 \mathrm{U} / \mathrm{ml}$ and SCF $8 \mathrm{U} / \mathrm{ml}$ (Genzyme Cambridge, MA, USA) and the plates were incubated in humidified air with $5 \% \mathrm{CO}_{2}$ at $37^{\circ} \mathrm{C}$ for 14 days. The aggregates containing $>50$ cells were scored as colonies, whereas those containing $<50$ cells were scored as clusters. All of the cultures were set up in quadruplicate. In order to exclude interference due to the absence of reducing equivalents in the agar assay, clonogenic tests using methylcellulose (HF 4434, Stem Cell Technologies Vancouver, Canada) were also made in a subset of patients.

\subsection{TNF- $\alpha$ levels in BM plasma and liquid culture}

The plasma samples obtained by spinning the BM samples at $3000 \mathrm{rpm}$ were stored at $-20^{\circ} \mathrm{C}$ until used, and then investigated for their TNF- $\alpha$ content by means of a commercially available immunoenzymaticassay kit (Medgenix Diagnostic, Fleurus, Belgium). The TNF- $\alpha$ levels in the supernatants of $24 \mathrm{~h}$ liquid cultures were also evaluated. The minimum detectable dose of TNF- $\alpha$ was $3.0 \mathrm{pg} / \mathrm{ml}$.

\subsection{Statistical analysis}

The Wilcoxon matched pairs signed ranks test was used to evaluate within-group differences, and the Mann-Whitney test for differences between groups. A $P$ value of $<0.05$ was considered to be statistically significant. 


\section{Results}

4.1. Evaluation of apoptosis in fresh bone marrow mononuclear cells (BMMNCs)

The apoptosis evaluated in fresh MDS BMMNCs was higher than that observed in the normal samples $(1.67 \pm 0.16$ SE versus $1.49 \pm 0.27$, n.s.). Apoptosis was even higher in the LR group (LR: $2.02 \pm 0.22, P<0.05$ versus normals).

\subsection{Evaluation of apoptosis after $24 h$ of incubation with NAC}

In the normal samples, apoptosis was significantly $(P<0.05)$ less after NAC incubation in comparison with controls $(3.03 \pm 0.74$ versus $4.1 \pm 0.84)$. It was also less after NAC stimulation in the MDS samples as a whole $(2.12 \pm 0.52$ versus $3.44 \pm 0.66)(P<0.01)$, as well as in the two subgroups (LR: $2.06 \pm 0.68$ versus $3.67 \pm 0.93$; HR: $2.18 \pm 0.83$ versus $3.16 \pm 0.96)(P<$ 0.05 and $P<0.01$, respectively) (Fig. 1).

\subsection{Clonogenic assay (CFU-GM) after $24 \mathrm{~h}$ of incubation with $N A C$}

The number of CFU-GM was significantly higher in the NAC treated normal than in the controls (195.6 \pm 49.72 versus $153.8 \pm 45.98)(P<0.05)$. A significant increase in the number of CFU-GM was also observed in the MDS patients after NAC incubation (MDS: $73.39 \pm 12.88$ versus $57.3 \pm 11.24, \quad P<0.01 ; \quad$ LR: $78.69 \pm 14.59$ versus $61.85 \pm 13.79, \quad P<0.01 ; \quad$ HR: $66.5 \pm 23.54$ versus $51.4 \pm 19.34, P<0.05$ ) (Fig. 2).
4.4. Evaluation of apoptosis after 48 h of incubation with NAC \pm ATRA

ATRA alone did not modify the percentage of apoptosis in either the normals or MDS samples, but incubation with NAC + ATRA led to a significant decrease in the apoptotic rate in both the normal $(2.36 \pm 0.61$ versus $3.74 \pm 0.97, P<0.05)$ and MDS samples $(2.06 \pm$ 0.44 versus $3.78 \pm 0.7, P<0.01)$. The same was observed in both the LR $(P<0.05)$ and HR $(P=0.06)$ subgroups (LR: $1.62 \pm 0.34$ versus $3.3 \pm 0.69$; HR: $2.71 \pm 0.96$ versus $4.36 \pm 1.23$ ) (Fig. 3). Apoptosis was also less in the 12 MDS samples evaluated after $48 \mathrm{~h}$ NAC stimulation than in controls $(1.52 \pm 0.85$ versus $3.16 \pm 1.08, P<0.05$ ) (Fig. 3a).

\subsection{Clonogenic assay (CFU-GM) after $48 \mathrm{~h}$ of incubation with NAC \pm ATRA}

Both ATRA and NAC + ATRA significantly increased clonogenic activity in both the normal $(P<$ $0.05)$ and MDS samples $(P<0.01)$ (normal: CTRL $172.2 \pm 36.06$, ATRA $329.8 \pm 62.56, \quad \mathrm{NAC}+\mathrm{ATRA}$ $361 \pm 65.29$; $\quad$ MDS: $\quad$ CTRL $59.67 \pm 15.28$, ATRA $74.76 \pm 14.95, \mathrm{NAC}+$ ATRA $87.59 \pm 19.56$ ).

However although incubation with ATRA alone increased clonogenic activity in both MDS subgroups, this was statistically significant only in the LR patients $(P<0.01)$; the increase after incubation with NAC + ATRA was statistically significant in both the LR $(P<0.01)$ and HR patients $(P<0.05)$ (mean values LR: CTRL 78.58 \pm 21.83 , ATRA $100.67 \pm 21.64$, NAC + ATRA $110.08 \pm 29.06$; HR: CTRL $34.44 \pm$ 18.7, ATRA $40.22 \pm 13.59$, NAC + ATRA $55.11 \pm$

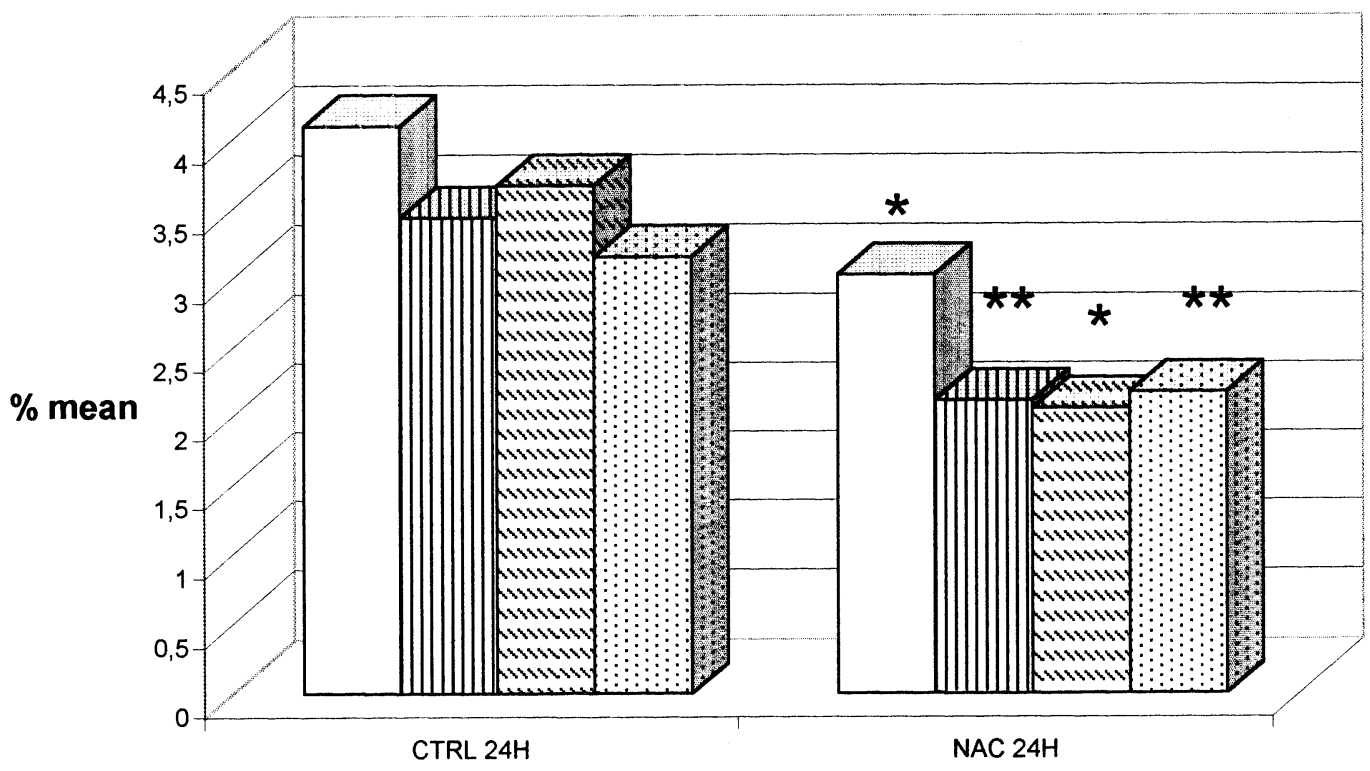

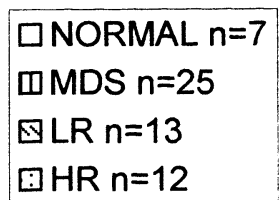

Fig. 1. Effect of NAC on apoptosis after $24 \mathrm{~h}$ of liquid culture. ${ }^{*} P<0.05,{ }^{* *} P<0.01$ versus controls. 


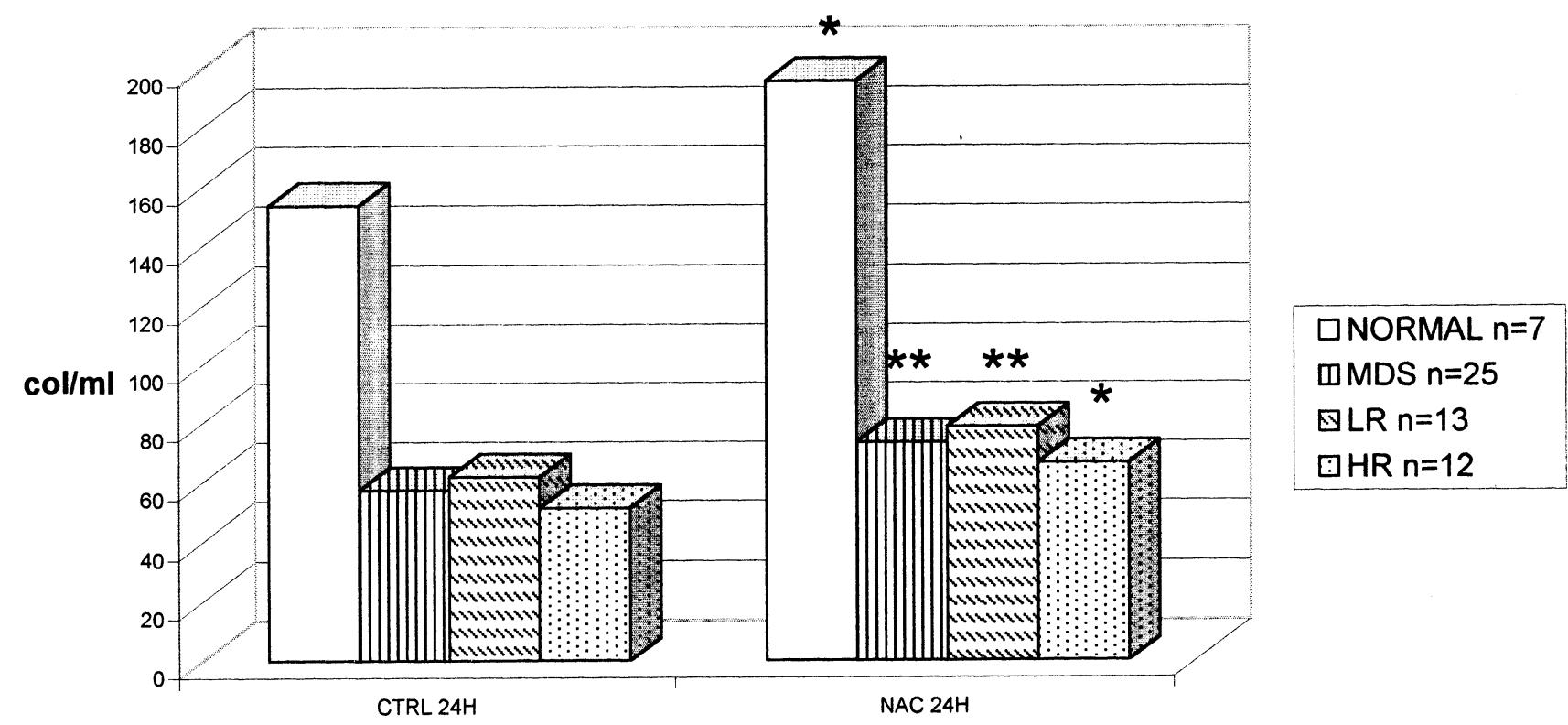

Fig. 2. Effect of NAC on clonogenic activity (CFU-GM) after $24 \mathrm{~h}$ of liquid culture. ${ }^{*} P<0.05, * * P<0.01$ versus controls.

20.09) (Fig. 4). The NAC + ATRA combination conferred a significant $(P<0.05)$ advantage in terms of clonogenic potentials with respect to ATRA in the MDS samples as a whole.

After $48 \mathrm{~h}$ incubation with NAC alone, the increase in the clonogenic activity of the MDS samples was statistically significant $(61.5 \pm 12.63$ versus $42.75 \pm$ $10.48, P<0.05)$ in comparison with the control samples, but significantly $(P<0.05)$ less than that observed after incubation with NAC + ATRA $(69 \pm 13.61)$ (Fig. 4a).

Analysis of the colony/cluster ratio after 24 and $48 \mathrm{~h}$ always revealed a moderate and non-significant increase in the MDS samples as a whole (24 h: CTRL $29.42 \pm$ 11.48 , NAC $35.99 \pm 12.92$; 48 h: CTRL $24.86 \pm 8.38$, ATRA $37.35 \pm 12.89$, NAC $32.83 \pm 16.33$, NAC + ATRA $37.27 \pm 10.91)$ as well as in the LR (24 h: CTRL $45.93 \pm 20.41, \quad$ NAC $56.06 \pm 20.46 ; \quad 48$ h: CTRL $37.13 \pm 13.84$, ATRA $54.98 \pm 21.04$, NAC $52.03 \pm$ 29.12, NAC + ATRA $49.49 \pm 16.17$ ) and HR patients (24 h: CTRL $11.08 \pm 4.98$, NAC $13.69 \pm 6.66 ; 48$ h: CTRL $11.05 \pm 6.55, \quad$ ATRA $17.52 \pm 11.46, \quad$ NAC $13.63 \pm 8.81, \mathrm{NAC}+$ ATRA $22 \pm 13.02)$. We never observed a reduction in the colony/cluster ratio of any patient sample or under any culture conditions.

The data obtained using methylcellulose assay were comparable to those derived from agar assay (data not shown).

\subsection{TNF- $\alpha$ levels in BM plasma and liquid culture}

BM plasma TNF- $\alpha$ levels were significantly higher $(P<0.01)$ in the MDS patients $(36.94 \pm 3.58$, range $15.53-70.81 \mathrm{pg} / \mathrm{ml})$ than in the normal subjects $(8.38 \pm$
0.44 , range $3.06-13.77 \mathrm{pg} / \mathrm{ml})$. No difference was found between the LR and HR subgroups, although the highest TNF- $\alpha(70.81 \mathrm{pg} / \mathrm{ml})$ value was observed in a LR patient (Fig. 5). However, the patients with TNF- $\alpha$ levels of $>50 \mathrm{pg} / \mathrm{ml}$ also responded to NAC in terms of CFU-GM and apoptosis.

The TNF- $\alpha$ levels in the supernatants of $24 \mathrm{~h} \mathrm{BM}$ MDS liquid cultures showed a significant $(P<0.05)$ decrease after incubation with NAC $(16.99 \pm 6.22)$ in comparison with the control samples $(26.72 \pm 5.78)$ (Fig. 6).

\section{Discussion}

The apparently bizarre behaviour of MDS hematopoiesis, which is expressed in the form of peripheral cytopenia associated with normo-hypercellular bone marrow, is the consequence of increased apoptosis and the maturation arrest of MDS hemopoietic progenitors. It is also known that high levels of TNF- $\alpha$ (a pro-apoptotic agent) are found in the serum of MDS patients, and so the documented oxidative damage to MDS $\mathrm{CD} 34+$ cells could also be the result of TNF- $\alpha$ induced free radical production.

On the basis of these observations, we evaluated the effect of an antioxidant (and anti-apoptotic) agent (NAC) and a differentiating agent (ATRA), alone and in association, on clonogenic activity and apoptosis in MDS BM mononuclear cells.

Our study showed the in vitro ability of NAC to enhance hemopoiesis in MDS, even in the presence of a large percentage of blasts. After 24 and $48 \mathrm{~h}$ of incubation with NAC, we observed an increase in the number 
of CFU-GM in the MDS samples as a whole, as well as in the LR and HR groups. A consensual decrease in the apoptotic rate was seen after 24 and $48 \mathrm{~h}$ NAC stimulation. These data indirectly suggest that apoptosis plays a role even in the MDS HR subset. Moreover, ATRA did not have any adverse effects on erythroid colonies, evaluated in methylcellulose assay (data not shown). NAC alone, in fact, slightly enhanced the number of BFU-E colonies whereas the association ATRANAC neither increased nor decreased the number of colonies.

As a result of the physiological senescence of cultured cells, the apoptosis evaluated in the control samples (i.e. after incubation with medium alone) was higher than that observed in the fresh samples. Moreover, the level of apoptosis was particularly high in the MDS HR subgroup. Since our observations are derived from a mixed population of all BMMNCs, we cannot be sure whether or not this phenomenon is due to CD34 + cells. However this singular behaviour may be explained by the poor self-renewal of MDS blasts, which are less capable of proliferation than leukemic blasts, as has previously been pointed out by Aul et al. [31]. A propensity towards apoptosis therefore seems to coexist with enhanced proliferation in HR MDS cells, and this may be responsible for the relative indolence of RAEB and RAEB-t.

The efficacy of NAC may be due to an increase in intracellular glutathione (GSH) levels in MDS CD34+ cells, which in turn provides protection against the apoptosis induced by free radicals. Furthermore, NAC may also prevent the release of TNF- $\alpha$ from the lymphocytes still present in BM liquid cultures, a hypothesis that is apparently supported by the decreased
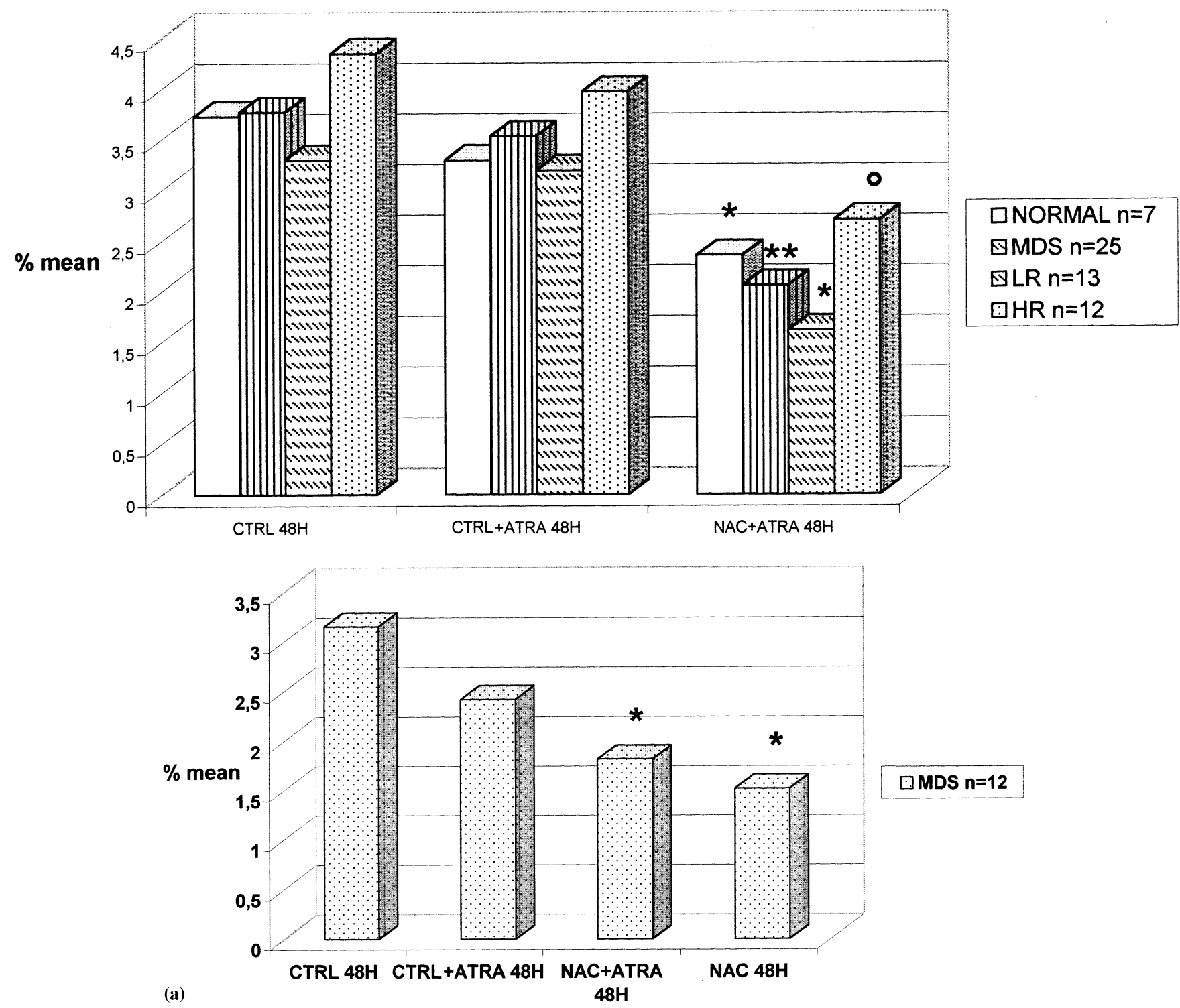

Fig. 3. Effect of NAC and/or ATRA on apoptosis after $48 \mathrm{~h}$ of liquid culture. ${ }^{*} P<0.05,{ }^{* *} P<0.01,{ }^{\circ} P=0.06$ versus controls. 


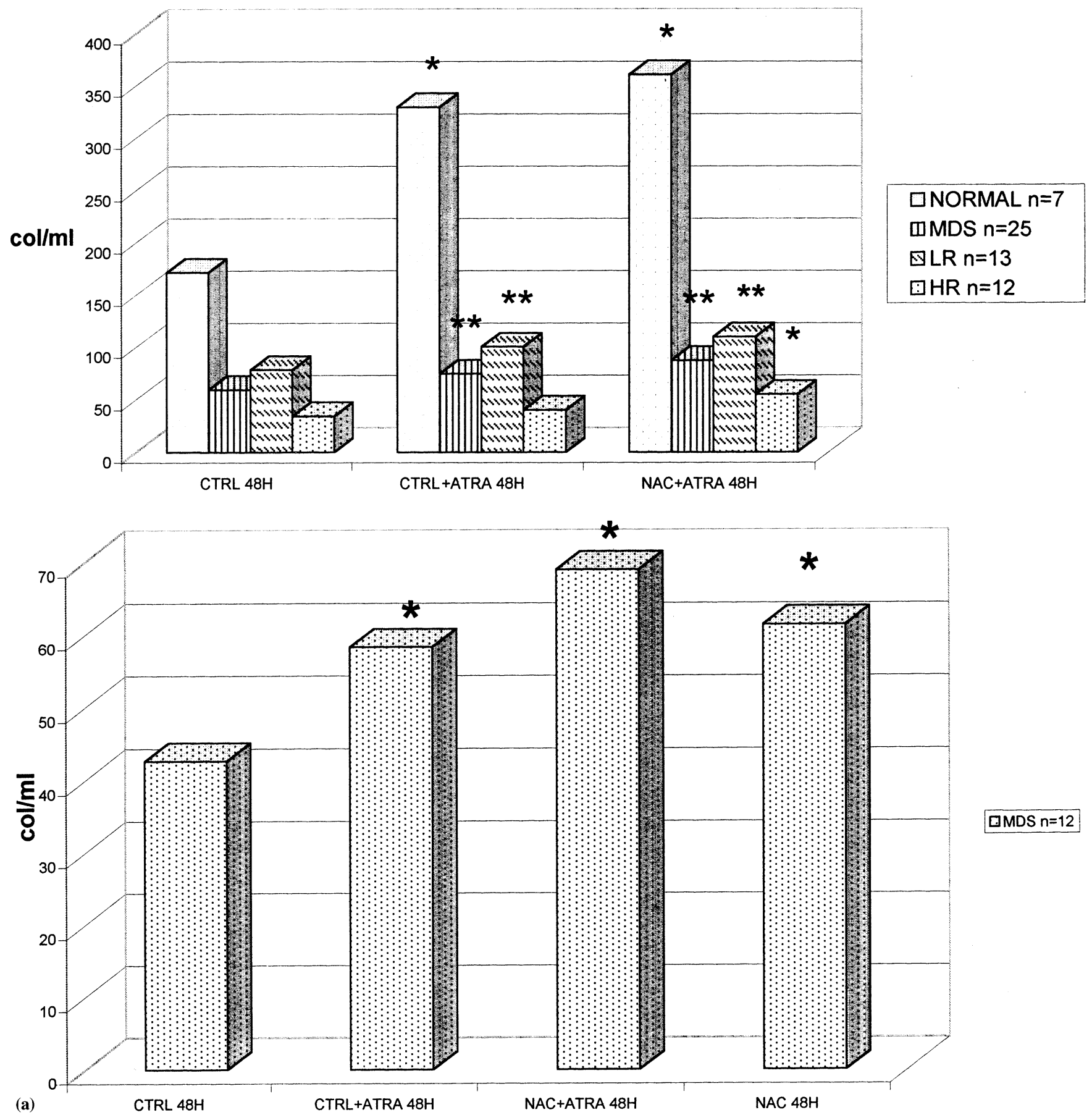

(a) CTRL $48 \mathrm{H}$

CTRL+ATRA 48H

NAC+ATRA 48H

$\mathrm{NAC} 48 \mathrm{H}$

Fig. 4. Effect of NAC and/or ATRA on clonogenic activity (CFU-GM) after $48 \mathrm{~h}$ of liquid culture. ${ }^{*} P<0.05$, $* * P<0.01$ versus controls.

release of TNF- $\alpha$ in the supernatant of the liquid cultures. NAC was also effective in the patients with the highest TNF- $\alpha$ levels.

On the contrary, and as expected, ATRA did not modify the apoptotic rate of MDS or normal BM cells, and a significantly enhanced clonogenic activity was only observed in the MDS samples as a whole and in the LR sub-group, thus suggesting that only patients with a low degree of BM blasts retain differentiative ability. The fact that, in all of the MDS samples, the
$\mathrm{NAC}+$ ATRA combination seemed to confer an advantage over the use of either substances alone in terms of clonogenic activity suggests that anti-oxidant treatment may play a key role in guaranteeing MDS cell survival and predisposing them towards differentiation. There was no evidence that NAC or ATRA add any leukemogenic effect on MDS BM cells, as the number of clusters remained the same after stimulation with either substance, whereas the number of colonies always increased. 


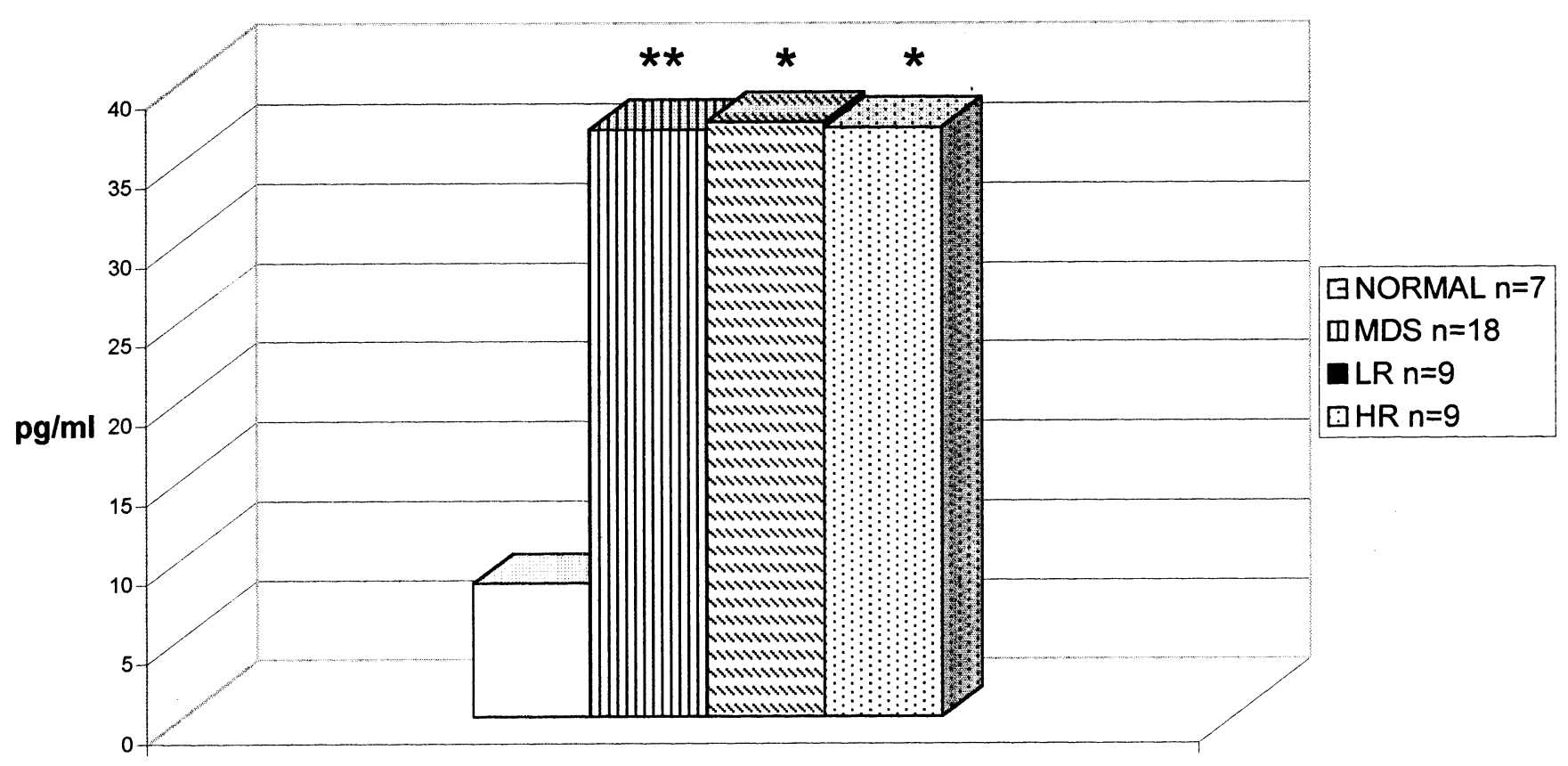

Fig. 5. Bone marrow plasma TNF- $\alpha$ levels. ${ }^{*} P<0.05, * * P<0.01$ versus controls.

It has recently been reported that combined therapy with ciprofloxacin, pentoxifylline and dexamethasone is efficacious in reducing TNF- $\alpha$ levels and improving hemopoiesis in MDS patients [9]. Moreover, List et al. [32] have demonstrated that the aminothiol amyfostine is capable of improving the in vitro clonogenic activity of MDS hemopoietic precursors. The in vivo results seem to be encouraging [33], although Bowen et al. [34] observed a poor response rate to amyfostine therapy. Our own in vitro results strongly suggest that a substance with a very low toxicity profile such as NAC could be included in the non-cytotoxic therapy of MDS.

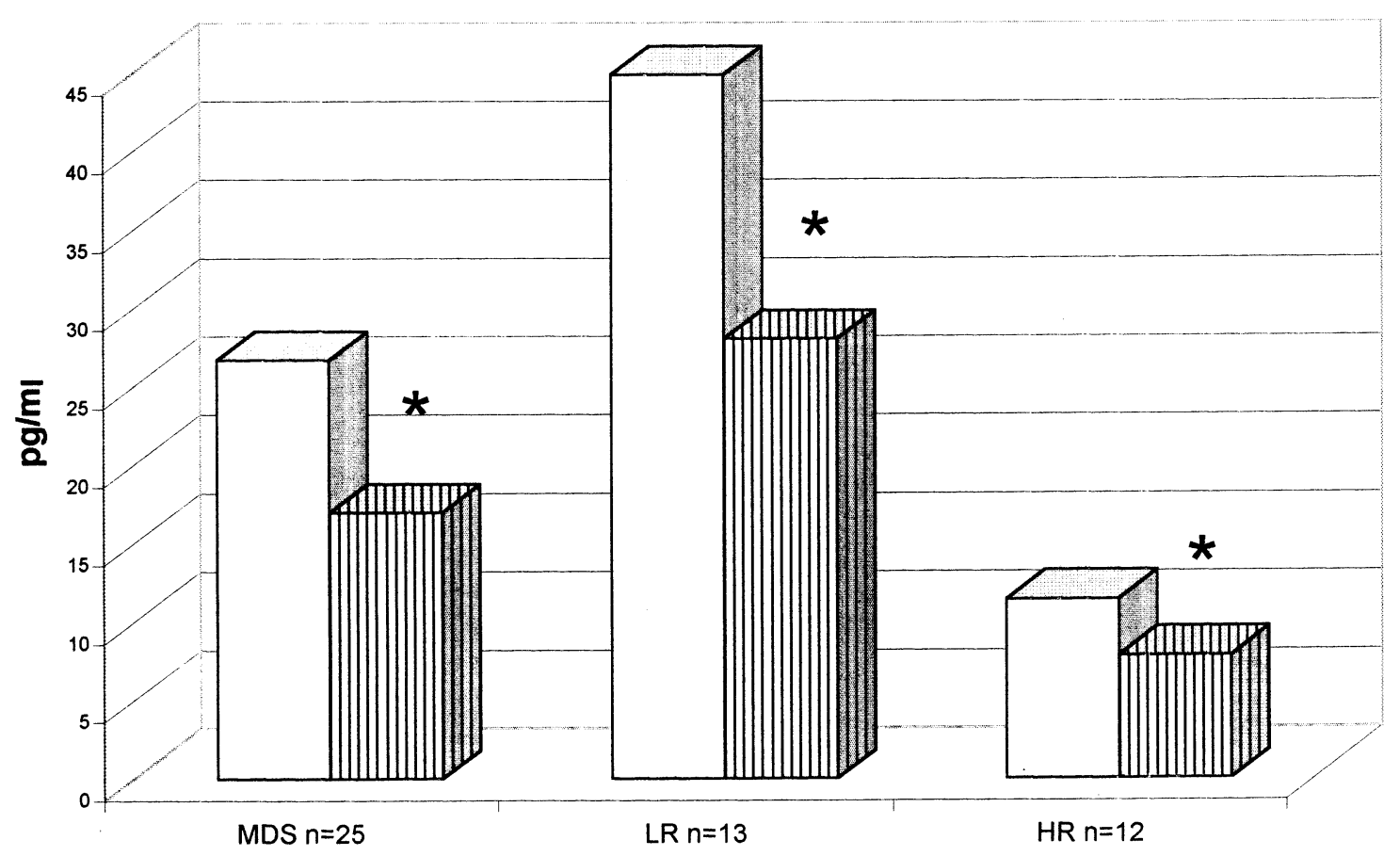

Fig. 6. TNF- $\alpha$ levels after $24 \mathrm{~h}$ cultures. ${ }^{*} P<0.05$ versus controls. 


\section{Acknowledgements}

A Cortelezzi provided the concept, design, drafting of the paper, critical revision and gave final approval. C Cattaneo provided statistical expertise and contributed to the concept design, drafting of the paper, critical revision. B Sarina provided statistical help, assisted in drafting the paper and gave final approval. S Cristiani assisted with data interpretation, provided statistical expertise, administrative support and helped with data assembly and gave final approval. M Pomati helped with analysis of the data. I Silvestris collected and helped with the data analysis. M Motta provided study materials and helped to assemble the data. A Ibatici provided study materials. AT Maiola provided funding for the project.

\section{References}

[1] Hamblin TJ, Oscier DG. The myelodysplastic syndrome - a practical guide. Hematol Oncol 1987;5:19-34.

[2] Oscier DG. Myelodysplastic syndromes. Baillieres Clin Haematol 1987;1:389-426.

[3] Yoshida Y. Hypotesis: apoptosis may be the mechanism responsible for the premature intramedullary cell death in myelodysplastic syndrome. Leukemia 1993;7:144-6.

[4] Raza A, Gezer S, Mundle S, Gao XZ, Alvi S, Borok R, et al. Apoptosis in bone marrow biopsy samples involving stromal and hematopoietic cells in 50 patients with myelodysplastic syndromes. Blood 1995;86:268-76.

[5] Yoshida Y, Anzai N, Kawabata H. Apoptosis in myelodysplasia: a paradox or paradigm. Leuk Res 1995;19:887-91.

[6] Rajapaksa R, Ginzton N, Rott LS, Greenberg PL. Altered oncoprotein expression and apoptosis in myelodysplastic syndrome marrow cells. Blood 1996;88:4275-87.

[7] Verhoef GE, De Schouwer P, Ceuppens JL, Van Damme J, Goossens W, Boogaerts MA. Measurement of serum cytokine levels in patients with myelodysplastic syndromes. Leukemia 1992;6:1268-72.

[8] Gersuk GM, Beckham C, Loken MR, Kiener P, Anderson JE, Farrand A, et al. A role for tumor necrosis factor-alfa, Fas and Fas-ligand in marrow failure associated with myelodysplastic syndrome. Br J Haematol 1998;103:176-88.

[9] Reza S, Shetty V, Dar S, Qawi H, Raza A. Tumor necrosis factor-alfa levels decrease with anticytokine therapy in patients with myelodysplastic syndromes. J Interferon Cytokine Res 1998;18:871-7.

[10] Raza A, Mundle S, Shetty V, Alvi S, Chopra H, Span L, et al. Novel insights into the biology of myelodysplastic syndromes: excessive apoptosis and the role of cytokines. Int $\mathbf{J}$ Hematol 1996;63:265-78

[11] Raza A, Mundle S, Shetty V, Alvi S, Chopra H, Span L, et al. A paradigm shift in myelodyslastic syndromes. Leukemia 1996;10:1648-52.

[12] Shetty V, Mundle S, Alvi S, Showel M, Broady-Robinson L, Dar S, et al. Measurement of apoptosis, proliferation and three cytokines in 46 patients with myelodysplastic syndromes. Leuk Res 1996;20:891-900.

[13] Shoji Y, Uedono Y, Ishikura UH, Takeyama N, Tanaka T. DNA damage induced by tumor necrosis factor- $\alpha$ in L929 cells is mediated by mitochondrial oxygen radical formation. Immunology $1995 ; 84: 543-8$.
[14] Beg A, Baltimore D. An essential role for NF-kB in preventing TNF- $\alpha$-induced cell death. Science 1996;274:782-4.

[15] Wang CY, Mayo MW, Baldwin Jr AS. TNF and cancer therapy-induced apoptosis: potentiation by inhibition of NF-kB. Science 1996;274:784-7.

[16] Jabbar SA, Hoffbrand AV, Wickremasinghe G. Redox reagents and staurosporine inhibit stimulation of the transcription regulator NF-kB following tumor necrosis factor treatment of chronic B-leukaemia cells. Leuk Res 1994;18:523-30.

[17] Peddie CM, Wolf CR, McLellan LI, Collins AR, Bowen DT. Oxidative DNA damage in CD34+ myelodysplastic cells is associated with intracellular redox changes and elevated plasma tumor necrosis factor- $\alpha$ concentration. $\mathrm{Br} \quad \mathrm{J}$ Haematol 1997;99:625-31.

[18] Talley AK, Dewhurst S, Perry SW, Dollard SC, Gummuluru S, Fine SM, et al. Tumor necrosis factor alpha-induced apoptosis in human neuronal cells: protection by the antioxidant $N$-acetylcysteine and the genes bcl2 and crmA. Mol Cell Biol 1995; 15:2359-66.

[19] Cossarizza A, Franceschi C, Monti D, Salvioli S, Bellesia E, Rivabene R, et al. Protective effect of $N$-acetylcysteine in tumor necrosis factor- $\alpha$-induced apoptosis in U937 cells: the role of mitochonria. Exp Cell Res 1995;220:232-40.

[20] Mayer M, Noble M. $N$-acetylcysteine is a pluripotent protector against cell death and enhancer of trophic factor-mediated cell survival in vitro. Proc Natl Acad Sci 1994;91:7496-500.

[21] Delneste Y, Jeannin P, Potier L, Romero P, Bonnefoy JY. $N$-acetylcysteine exhibits antitumoral activity by increasing tumor necrosis factor- $\alpha$-dependent T-cell-cytotoxicity. Blood 1997;90:1124-32.

[22] Santini V. Differentiation theory of myelodisplastic syndromes: fact or fiction? Br J Haematol 1998;102:1124-38.

[23] Tallman MS. Differentiating therapy in acute myeloid leukemia. Leukemia 1996;10(Suppl. 2):S33-8.

[24] Ohno R. Differentiation therapy of myelodisplastic syndromes with retinoic acid. Leuk Lymphoma 1994;14:401-9.

[25] Venditti A, Stasi R, Del Poeta G, Buccisano F, Aronica G, Bruno A, et al. All-trans retinoic acid and low-dose cytosine arabinoside for the treatment of 'poor prognosis' acute myeloid leukemia. Leukemia 1995;9:1121-5.

[26] Aul C, Runde V, Gattermann N. All-trans-retinoic acid in patients with myelodysplastic syndromes: results of a pilot study. Blood 1993;82:2967-74.

[27] Ohno R, Naoe T, Hirano M, Kobayashi M, Hirai H, Tubaki K, et al. Treatment of myelodysplastic syndromes with all-transretinoic acid. Blood 1993;81:1152-4.

[28] Visani G, Tosi P, Manfroi E, Ottaviani E, Finelli C, Cenacchi A, et al. All-trans-retinoic acid in the treatment of myelodysplastic syndromes. Leuk Lymphoma 1995;19:277-80.

[29] Ganser A, Maurer A, Contzen C, Seipelt G, Ottmann OG, Schadeck-Gressel C, et al. Improved multilineage response of hematopoiesis in patients with myelodysplastic syndromes to a combination therapy with all-trans-retinoic acid, granulocyte colony-stimulating factor, erythropoietin and $\alpha$-tocopherol. Ann Hematol 1996;72:237-44.

[30] Ganser A, Seipelt G, Verbeek W, Ottmann OG, Maurer A, Kolbe $\mathrm{K}$, et al. Effects of combination therapy with all-transretinoic acid and recombinant human granulocyte colony-stimulating factor in patients with myelodysplastic syndromes. Leukemia 1994;8:369-75.

[31] Aul C, Gattermann N, Schneider W. Comparison of in vitro growth characteristics of blast cell progenitors (CFU-L) in patients with myelodisplastic syndromes and acute myeloid leukemia. Blood 1992;80:625-33.

[32] List AF, Heaton R, Glinsmann-Gibson B, Capizzi RL. Amifostine protects primitive hematopoietic progenitors against chemotherapy cytotoxicity. Semin Oncol 1996;23:58-61. 
[33] List AF, Brasfield F, Heaton R, Glinsmann-Gibson B, Crook L, Taetle R, et al. Stimulation of hematopioesis by amifostine in patients with myelodysplastic syndrome. Blood 1997;90:33649.
[34] Bowen DT, Dezlienger C, Brugger W, Culligan D, Gelly K, Adlakha S, et al. Poor response rate to a continuous schedule of Amifostine therapy for 'low/intermediate risk' myelodysplastic patients. Br J Haematol 1998;103:785-7. 